\title{
INEQUALITIES FOR DIFFERENTIABLE REPRODUCING KERNELS AND AN APPLICATION TO POSITIVE INTEGRAL OPERATORS
}

\author{
JORGE BUESCU AND A. C. PAIXÃO
}

Received 18 October 2005; Revised 7 November 2005; Accepted 13 November 2005

Let $I \subseteq \mathbb{R}$ be an interval and let $k: I^{2} \rightarrow \mathbb{C}$ be a reproducing kernel on $I$. We show that if $k(x, y)$ is in the appropriate differentiability class, it satisfies a 2-parameter family of inequalities of which the diagonal dominance inequality for reproducing kernels is the 0th order case. We provide an application to integral operators: if $k$ is a positive definite kernel on $I$ (possibly unbounded) with differentiability class $\mathscr{Y}_{n}\left(I^{2}\right)$ and satisfies an extra integrability condition, we show that eigenfunctions are $C^{n}(I)$ and provide a bound for its Sobolev $H^{n}$ norm. This bound is shown to be optimal.

Copyright (c) 2006 J. Buescu and A. C. Paixão. This is an open access article distributed under the Creative Commons Attribution License, which permits unrestricted use, distribution, and reproduction in any medium, provided the original work is properly cited.

\section{Introduction}

Given a set $E$, a positive definite matrix in the sense of Moore (see, e.g., Moore $[5,6]$ and Aronszajn [1]) is a function $k: E \times E \rightarrow \mathbb{C}$ such that

$$
\sum_{i, j=1}^{n} k\left(x_{i}, x_{j}\right) \bar{\xi}_{i} \xi_{j} \geq 0
$$

for all $n \in \mathbb{N},\left(x_{1}, \ldots, x_{n}\right) \in E^{n}$ and $\left(\xi_{1}, \ldots, \xi_{n}\right) \in \mathbb{C}^{n}$; that is, all finite square matrices $M$ of elements $m_{i j}=k\left(x_{i}, x_{j}\right), i, j=1, \ldots, n$, are positive semidefinite.

From (1.1) it follows that a positive definite matrix in the sense of Moore has the following basic properties: (1) it is conjugate symmetric, that is, $k(x, y)=\overline{k(y, x)}$ for all $x, y \in E,(2)$ it satisfies $k(x, x) \geq 0$ for all $x \in E$, and (3) $|k(x, y)|^{2} \leq k(x, x) k(y, y)$ for all $x, y \in E$. We sometimes refer to this last basic inequality as the "diagonal dominance" inequality.

The theorem of Moore-Aronszajn [1, 5, 6] provides an equivalent characterization of positive definite matrices as reproducing kernels: $k: E \times E \rightarrow \mathbb{C}$ is a positive definite matrix in the sense of Moore if and only if there exists a (uniquely determined) Hilbert space $H_{k}$ 
2 Differential inequalities and integral operators

composed of functions on $E$ such that

$$
\begin{gathered}
\forall y \in E, \quad k(x, y) \in H_{k} \text { as a function of } x, \\
\forall x \in E \text { and any } f \in H_{k}, \quad f(x)=\langle f(y), k(y, x)\rangle_{H_{k}} .
\end{gathered}
$$

Properties (1.2) are jointly called the reproducing property of $k$ in $H_{k}$. The function $k$ itself is called a reproducing kernel on $E$ and the associated (and unique) Hilbert space $H_{k}$ a reproducing kernel Hilbert space; see, for example, Saitoh [8].

Throughout this paper we deal exclusively with the case where $E=I \subseteq \mathbb{R}$ is a real interval, nontrivial but otherwise arbitrary; in particular $I$ may be unbounded. Only in Section 3 we will need the further assumption that $I$ is closed; this extra condition will at that point be explicitly required. If $x \in I$ is a boundary point of $I$, a limit at $x$ will mean the one-sided limit as $y \rightarrow x$ with $y \in I$.

Definition 1.1. Let $I \subset \mathbb{R}$ be an interval. A function $k: I^{2} \rightarrow \mathbb{C}$ is said to be of class $\mathscr{Y}_{n}\left(I^{2}\right)$ if, for every $m_{1}=0,1, \ldots, n$ and $m_{2}=0,1, \ldots, n$, the partial derivatives $\partial^{m_{1}+m_{2}} / \partial y^{m_{2}} \partial x^{m_{1}} k(x$, $y$ ) are continuous in $I^{2}$.

Remark 1.2. Clearly from the definition $C^{2 n}\left(I^{2}\right) \subset \mathscr{S}_{n}\left(I^{2}\right) \subset C^{n}\left(I^{2}\right)$. It is also clear that a function of class $\mathscr{T}_{n}\left(I^{2}\right)$ will not in general be in $C^{n+1}\left(I^{2}\right)$. Note however that in class $\mathscr{Y}_{n}\left(I^{2}\right)$ equality of all intervening mixed partial derivatives holds.

In [4, Theorem 2.7], the following result is shown to hold for differentiable reproducing kernels as a nontrivial consequence of positive semidefiniteness of the matrices $k\left(x_{i}, x_{j}\right)$ in $(1.1)$.

Theorem 1.3. Let $I \subset \mathbb{R}$ be an interval and let $k(x, y)$ be a reproducing kernel on $I$ of class $\mathscr{S}_{n}\left(I^{2}\right)$. Then for all $x, y \in I$ and all $0 \leq m \leq n$,

$$
\left|\frac{\partial^{m} k}{\partial x^{m}}(x, y)\right|^{2} \leq \frac{\partial^{2 m} k}{\partial y^{m} \partial x^{m}}(x, x) k(y, y) .
$$

Remark 1.4. An immediate consequence of conjugate symmetry of $k$ is that inequality (1.3) is equivalent to

$$
\left|\frac{\partial^{m} k}{\partial y^{m}}(x, y)\right|^{2} \leq \frac{\partial^{2 m} k}{\partial y^{m} \partial x^{m}}(y, y) k(x, x) .
$$

Remark 1.5. Observe that the 1-parameter family of inequalities (1.3) coupled with the condition $k(y, y) \geq 0$ for all $y \in I$ implies that

$$
\frac{\partial^{2 m} k}{\partial y^{m} \partial x^{m}}(x, x) \geq 0
$$

for all $x \in I$ and all $0 \leq m \leq n$.

\section{Differentiable reproducing kernel inequalities}

Let $I \subseteq \mathbb{R}$ be an interval and $k: I \times I \rightarrow \mathbb{C}$. Denote by $I_{R}$ the set of all $x \in I$ such that $x+h$ is in $I$ for $|h|<R$. For sufficiently small $R, I_{R}$ is a nonempty open interval. For $|h|<R$ we 
define $\delta_{h}: I_{R}^{2} \rightarrow \mathbb{C}$ by

$$
\delta_{h}(x, y)=k(x+h, y+h)-k(x+h, y)-k(x, y+h)+k(x, y) .
$$

We then have the following lemma.

LEMmA 2.1. If $k(x, y)$ is a reproducing kernel on $I^{2}$ and $|h|<R$, then $\delta_{h}(x, y)$ is a reproducing kernel in $I_{R}^{2}$.

Proof. Let $l \in \mathbb{N},\left(x_{1}, \ldots, x_{l}\right) \in I_{h}^{l}$ and $\left(\xi_{1}, \ldots, \xi_{l}\right) \in \mathbb{C}^{l}$. We are required to show that $\sum_{i, j=1}^{l} \delta_{h}\left(x_{i}, x_{j}\right) \xi_{i} \bar{\xi}_{j} \geq 0$. Define $x_{l+i}=x_{i}+h$ and $\xi_{l+i}=-\xi_{i}$ for $i=1, \ldots, l$. Since $k$ is a reproducing kernel on $I^{2}$, we have $\sum_{i, j=1}^{2 l} k\left(x_{i}, x_{j}\right) \xi_{i} \overline{\xi_{j}} \geq 0$. Rewriting the left-hand side, we obtain

$$
\begin{aligned}
\sum_{i, j=1}^{2 l} k\left(x_{i}, x_{j}\right) \xi_{i} \bar{\xi}_{j}= & \sum_{i, j=1}^{l} k\left(x_{i}, x_{j}\right) \xi_{i} \overline{\xi_{j}} \\
& +\sum_{i=1}^{l} \sum_{j=l+1}^{2 l} k\left(x_{i}, x_{j}\right) \xi_{i} \overline{\xi_{j}}+\sum_{i=l+1}^{2 l} \sum_{j=1}^{l} k\left(x_{i}, x_{j}\right) \xi_{i} \overline{\xi_{j}}+\sum_{i, j=l+1}^{2 l} k\left(x_{i}, x_{j}\right) \xi_{i} \overline{\xi_{j}} \\
= & \sum_{i, j=1}^{l} k\left(x_{i}, x_{j}\right) \xi_{i} \overline{\xi_{j}}+\sum_{i, j=1}^{l} k\left(x_{i}, x_{j}+h\right) \xi_{i}\left(-\overline{\xi_{j}}\right)+\sum_{i, j=1}^{l} k\left(x_{i}+h, x_{j}\right)\left(-\xi_{i}\right) \overline{\xi_{j}} \\
& +\sum_{i, j=1}^{l} k\left(x_{i}+h, x_{j}+h\right)\left(-\xi_{i}\right)\left(-\overline{\xi_{j}}\right) \\
= & \sum_{i, j=1}^{l}\left[k\left(x_{i}+h, x_{j}+h\right)-k\left(x_{i}+h, x_{j}\right)-k\left(x_{i}, x_{j}+h\right)+k\left(x_{i}, x_{j}\right)\right] \xi_{i} \bar{\xi}_{j} \\
= & \sum_{i, j=1}^{l} \delta_{h}\left(x_{i}, x_{j}\right) \xi_{i} \overline{\xi_{j}} \geq 0 .
\end{aligned}
$$

Thus $\delta_{h}(x, y)$ is a reproducing kernel on $I_{R}^{2}$ as stated.

We will frequently denote, for ease of notation, $k_{m}(x, y)=\left(\partial^{2 m} k / \partial y^{m} \partial x^{m}\right)(x, y)$.

Proposition 2.2. Let $I \subset \mathbb{R}$ be an interval and let $k(x, y)$ be a reproducing kernel of class $\mathscr{Y}_{n}\left(I^{2}\right)$. Then, for all $0 \leq m \leq n, k_{m}(x, y)=\left(\partial^{2 m} / \partial y^{m} \partial x^{m}\right) k(x, y)$ is a reproducing kernel of class $\mathscr{S}_{n-m}\left(I^{2}\right)$.

Proof. Since in the case $n=0$ the statement is empty, we begin by concentrating on the case $m=n=1$. Suppose $k$ is of class $\mathscr{S}_{1}\left(I^{2}\right)$. Then, by [4, Lemma 2.5], if $|h|<R$, we have

$$
k_{1}(x, y)=\lim _{h \rightarrow 0} \frac{\delta_{h}(x, y)}{h^{2}}
$$

for every $(x, y) \in I_{R}^{2}$. By Lemma $2.1, \delta_{h}(x, y)$ is a reproducing kernel on $I_{R}^{2}$. Hence the last 
4 Differential inequalities and integral operators

inequality in (2.2) implies that

$$
\sum_{i, j=1}^{l} k_{1}\left(x_{i}, x_{j}\right) \xi_{i} \overline{\xi_{j}} \geq 0
$$

for any natural $l,\left(x_{1}, \ldots, x_{l}\right) \in I_{R}^{l}$ and $\left(\xi_{1}, \ldots, \xi_{l}\right) \in \mathbb{C}^{l}$. Therefore, $k_{1}(x, y)$ is a reproducing kernel on $I_{R}^{2}$. By continuity of $k_{1}$ inequality (2.4) holds for boundary points in $I_{2}$ (if they exist) with the interpretation of partial derivatives as appropriate one-sided limits. Thus (2.4) holds for all $\left(x_{1}, \ldots, x_{l}\right) \in I^{l}$ and every choice of $l \in \mathbb{N}$ and $\left(\xi_{1}, \ldots, \xi_{l}\right) \in \mathbb{C}^{l}$. Therefore $k_{1}$ is a reproducing kernel on $I^{2}$.

To conclude the proof, we now fix $n \in \mathbb{N}$, suppose that $k$ is a reproducing kernel of class $\mathscr{S}_{n}\left(I^{2}\right)$ and that $k_{m}$ is a reproducing kernel for some $m<n$. It is immediate to see that $k_{m}$ is of class $\mathscr{Y}_{n-m}\left(I^{2}\right)$. Repeating the argument used in the proof of the case $m=n=1$, we conclude that $k_{m+1}$ is a reproducing kernel. Therefore $k_{m}$ is a reproducing kernel for all $0 \leq m \leq n$. This finishes the proof.

Theorem 2.3. Let $I \subseteq \mathbb{R}$ be an interval and $k(x, y)$ be a reproducing kernel of class $\mathscr{S}_{n}\left(I^{2}\right)$. Then, for every $m_{1}, m_{2}=0,1, \ldots, n$ and all $x, y \in I$,

$$
\left|\frac{\partial^{m_{1}+m_{2}}}{\partial y^{m_{2}} \partial x^{m_{1}}} k(x, y)\right|^{2} \leq \frac{\partial^{2 m_{1}}}{\partial y^{m_{1}} \partial x^{m_{1}}} k(x, x) \frac{\partial^{2 m_{2}}}{\partial y^{m_{2}} \partial x^{m_{2}}} k(y, y) .
$$

Proof. Since $k$ is a reproducing kernel of class $\mathscr{S}_{n}\left(I^{2}\right)$, by Proposition $2.2 k_{m}$ is a reproducing kernel of class $\mathscr{S}_{n-m}\left(I^{2}\right)$ for every $0 \leq m \leq n$. Let $0 \leq m_{1} \leq m_{2} \leq n$. Then $k_{m_{1}}(x, y)=\left(\partial^{2 m_{1}} / \partial y^{m_{1}} \partial x^{m_{1}}\right) k(x, y)$ is a reproducing kernel of class $\mathscr{S}_{n-m_{1}}\left(I^{2}\right)$. We may write

$$
\begin{aligned}
\frac{\partial^{m_{1}+m_{2}}}{\partial y^{m_{2}} \partial x^{m_{1}}} k(x, y) & =\frac{\partial^{m_{2}-m_{1}}}{\partial y^{m_{2}-m_{1}}} \frac{\partial^{2 m_{1}}}{\partial y^{m_{1}} \partial x^{m_{1}}} k(x, y) \\
& =\frac{\partial^{m_{2}-m_{1}}}{\partial y^{m_{2}-m_{1}}} k_{m_{1}}(x, y) .
\end{aligned}
$$

Since $m_{2}-m_{1} \leq n-m_{1}$, application of Theorem 1.3 to $k_{m_{1}}$ yields

$$
\left|\frac{\partial^{m_{2}-m_{1}}}{\partial y^{m_{2}-m_{1}}} k_{m_{1}}(x, y)\right|^{2} \leq k_{m_{1}}(x, x) \frac{\partial^{2\left(m_{2}-m_{1}\right)}}{\partial y^{\left(m_{2}-m_{1}\right)} \partial x^{\left(m_{2}-m_{1}\right)}} k_{m_{1}}(y, y) .
$$

Hence

$$
\left|\frac{\partial^{m_{2}+m_{1}}}{\partial y^{m_{2}} \partial x^{m_{1}}} k(x, y)\right|^{2} \leq \frac{\partial^{2 m_{1}}}{\partial y^{m_{1}} \partial x^{m_{1}}} k(x, x) \frac{\partial^{2 m_{2}}}{\partial y^{m_{2}} \partial x^{m_{2}}} k(y, y)
$$

as stated. The proof of the case $0 \leq m_{2} \leq m_{1} \leq n$ can be obtained in a similar way using the corresponding inequalities derived by conjugate symmetry (see Remark 1.4).

Remark 2.4. Setting $n=0$ in Theorem 2.3 yields the statement that if the reproducing kernel $k(x, y)$ is continuous then the diagonal dominance inequality $|k(x, y)|^{2} \leq k(x$, $x) k(y, y)$ holds. Even though continuity is not necessary, this means that the diagonal 
dominance inequality for reproducing kernels may be thought of as the particular case $n=0$ in Theorem 2.3.

In this precise sense, Theorem 2.3 yields a 2-parameter family of inequalities which is the generalization of the diagonal dominance inequality for (sufficiently) differentiable reproducing kernels.

\section{Sobolev bounds for eigenfunctions of positive integral operators}

Throughout this section $I \subseteq \mathbb{R}$ will denote a closed, but not necessarily bounded, interval. A linear integral operator $K: L^{2}(I) \rightarrow L^{2}(I)$

$$
K(\phi)=\int_{I} k(x, y) \phi(y) d y
$$

with kernel $k(x, y) \in L^{2}\left(I^{2}\right)$ is said to be positive if

$$
\iint_{I} k(x, y) \overline{\phi(x)} \phi(y) d x d y \geq 0
$$

for all $\phi \in L^{2}(I)$. The corresponding kernel $k(x, y)$ is an $L^{2}(I)$-positive definite kernel. A positive definite kernel is conjugate symmetric for almost all $x, y \in I$, so the associated operator $K$ is self-adjoint. All eigenvalues of $K$ are real and nonnegative as a consequence of (3.2).

Definition 3.1. A positive definite kernel $k(x, y)$ in an interval $I \subseteq \mathbb{R}$ is said to be in class $\mathscr{A}_{0}(I)$ if

(1) it is continuous in $I^{2}$,

(2) $k(x, x) \in L^{1}(I)$

(3) $k(x, x)$ is uniformly continuous in $I$.

Remark 3.2. If $I$ is compact, the first condition trivially implies the other two, so $\mathscr{A}_{0}(I)$ coincides with the continuous functions $C\left(I^{2}\right)$. Definition 3.1 is therefore especially meaningful in the case where $I$ is unbounded. It has recently been shown [2] that, if $k$ is a positive definite kernel in class $\mathscr{A}_{0}(I)$, then the corresponding operator is compact, trace class and satisfies (the analog of) Mercer's theorem [7], irrespective of whether $I$ is bounded or unbounded. For this reason a positive definite kernel in class $\mathscr{A}_{0}(I)$ is sometimes called a Mercer-like kernel [4].

It may easily be shown [2] that, if $I$ is unbounded, the simultaneous conditions of $k(x, x) \in L^{1}(I)$ and uniform continuity of $k(x, x)$ in $I$ in Definition 3.1 may be equivalently replaced by $k(x, x) \in L^{1}(I)$ and $k(x, x) \rightarrow 0$ as $|x| \rightarrow+\infty$. This equivalent characterization of $\mathscr{A}_{0}(I)$ may sometimes be useful in applications (e.g., [3] or the proof of Theorem 3.5 below).

The following summarizes the properties of positive definite kernels relevant for this paper. If $k(x, y) \in L^{2}(I)$ is a positive definite kernel, then $K$ is a Hilbert-Schmidt operator; in particular it is compact, so its eigenvalues have finite multiplicity and accumulate only 
6 Differential inequalities and integral operators

at 0 . The spectral expansion

$$
k(x, y)=\sum_{i \geq 1} \lambda_{i} \phi_{i}(x) \overline{\phi_{i}(y)}
$$

holds, where the $\left\{\phi_{i}\right\}_{i \geq 1}$ are an $L^{2}(I)$-orthonormal set of eigenfunctions spanning the range of $K$, the $\left\{\lambda_{i}\right\}_{i \geq 1}$ are the nonzero eigenvalues of $K$ and convergence of the series (3.3) is in $L^{2}(I)$. If in addition $k$ is in class $\mathscr{A}_{0}(I)$, then for all $x \in I k(x, x) \geq 0$ and for all $x, y \in I|k(x, y)|^{2} \leq k(x, x) k(y, y)$, eigenfunctions $\phi_{i}$ associated to nonzero eigenvalues are uniformly continuous on $I$, convergence of the series (3.3) is absolute and uniform on $I$, and the operator $K$ is trace class and satisfies the trace formula $\int_{I} k(x, x) d x=\sum_{i \geq 1} \lambda_{i}$. In the case where $I$ is compact, the last statements are the classical theorem of Mercer; for proofs see, for example, [7] for compact $I$ and [2] for noncompact $I$. Finally, it is not difficult to show that continuous positive definite kernels are reproducing kernels on $I$ [4], so that the results of Section 2 apply.

Definition 3.3. Let $n \geq 1$ be an integer and $I \subseteq \mathbb{R}$. A positive definite kernel $k: I^{2} \rightarrow \mathbb{C}$ is said to belong to class $\mathscr{A}_{n}(I)$ if $k \in \mathscr{Y}_{n}(I)$ and

$$
k(x, y), \frac{\partial^{2} k}{\partial y \partial x}(x, y), \ldots, \frac{\partial^{2 n} k}{\partial y^{n} \partial x^{n}}(x, y)
$$

are in class $\mathscr{A}_{0}(I)$.

Remark 3.4. Trivially $\mathscr{A}_{n}(I) \subset \mathscr{A}_{n-1}(I) \subset \cdots \subset \mathscr{A}_{1}(I) \subset \mathscr{A}_{0}(I)$. More significantly, observe that a positive definite kernel in class $\mathscr{A}_{n}(I)$ possesses a delicate but precise mix of local (differentiability class $\mathscr{S}_{n}(I)$ ) and global (integrability and uniform continuity of each $k_{m}, m=0, \ldots, n$, along the diagonal $y=x$ ) properties.

For $k$ in class $\mathscr{A}_{n}(I)$, we set for each $m=0, \ldots, n$

$$
\mathcal{K}_{m} \equiv \int_{I} k_{m}(x, x) d x
$$

From Theorem 2.3 it follows that $0 \leq\left|k_{m}(x, y)\right|^{2} \leq k_{m}(x, x) k_{m}(y, y)$ for all $x, y \in I$. Thus for each $m=0, \ldots, n, \mathscr{K}_{m}>0$ unless $k_{m}(x, y)$ is identically zero. In the result below $H^{n}(I)$ denotes, as usual, the Sobolev Hilbert space $W^{n, 2}(I)$ normed by $\|\phi\|_{H^{n}(I)}^{2}=$ $\sum_{m=0}^{n}\left\|\phi^{(m)}\right\|_{L^{2}(I)}^{2}$. For $0 \leq l \leq n$, we define

$$
C_{n, l}=\mathscr{K}_{l}^{1 / 2}\left(\sum_{m=l}^{n} \mathscr{K}_{m}\right)^{1 / 2} .
$$

Theorem 3.5. Suppose $k(x, y)$ is a positive definite kernel in class $\mathscr{A}_{n}(I)$. Let $0 \leq l \leq n$ and let $\phi_{i}^{[l]}$ be a normalized eigenfunction of $k_{l}(x, y)$ associated with a nonzero eigenvalue $\lambda_{i}^{[l]}$. Then $\phi_{i}^{[l]}$ is in $C^{n-l}(I) \cap H^{n-l}(I)$ and

$$
\left\|\phi_{i}^{[l]}\right\|_{H^{n-l}(I)} \leq \frac{C_{n, l}}{\lambda_{i}^{[l]}} .
$$


Proof. Let $k$ be in $\mathscr{A}_{n}(I)$. Then $k_{l}$ is in $\mathscr{A}_{n-l}(I)$. For fixed $l=0, \ldots, n$, suppose $\phi_{i}^{[l]}$ is a normalized eigenfunction of $k_{l}$ associated to $\lambda_{i}^{[l]} \neq 0$, that is

$$
\phi_{i}^{[l]}(x)=\frac{1}{\lambda_{i}^{[l]}} \int_{I} k_{l}(x, y) \phi_{i}^{[l]}(y) d y
$$

with $\left\|\phi_{i}^{[l]}\right\|_{L^{2}(I)}=1$. In the case where $I$ is compact, differentiation of (3.8) $n-l$ times under the integral sign holds automatically, and so eigenfunctions are $C^{n-l}(I)$. For unbounded $I$ this is no longer automatic. We will show, however, that in this case it is also true, but as specific consequence of $k$ being a positive definite kernel in class $\mathscr{A}_{n}(I)$. Thus for the rest of the proof of the first statement $I$ will, without loss of generality, be taken to be $\mathbb{R}$.

By hypothesis, for $0 \leq l \leq m \leq n$ the integrand function $\left(\partial^{m-l} k_{l}(x, y)\right) /\left(\partial x^{m-l}\right) \phi_{i}^{[l]}(y)$ corresponding to the $(m-l)$ th differentiation under the integral sign exists and is continuous. We have

$$
\begin{aligned}
\left|\frac{\partial^{m-l}}{\partial x^{m-l}} k_{l}(x, y) \phi_{i}^{[l]}(y)\right| & =\left|\frac{\partial^{m-l}}{\partial x^{m-l}} k_{l}(x, y)\right|\left|\phi_{i}^{[l]}(y)\right| \\
& \leq\left(\frac{\partial^{2(m-l)}}{\partial y^{m-l} \partial x^{(m-l)}} k_{l}(x, x)\right)^{1 / 2} k_{l}(y, y)^{1 / 2}\left|\phi_{i}^{[l]}(y)\right| \\
& \leq k_{m}(x, x)^{1 / 2} k_{l}(y, y)^{1 / 2}\left|\phi_{i}^{[l]}(y)\right|
\end{aligned}
$$

where we have used Theorem 2.3 with $m_{1}=m-l, m_{2}=0$, and $k$ replaced with $k_{l}$. The fact that $k_{l}(y, y)^{1 / 2}\left|\phi_{i}^{[l]}(y)\right|$ is in $L^{1}(I)$ follows from the Cauchy-Schwartz inequality since

$$
\begin{aligned}
\int_{I} k_{l}(y, y)^{1 / 2}\left|\phi_{i}^{[l]}\right| d y & \leq\left(\int_{I} k_{l}(y, y) d y\right)^{1 / 2}\left\|\phi_{i}^{[l]}\right\|_{L^{2}(I)} \\
& =\left(\int_{I} k_{l}(y, y) d y\right)^{1 / 2}=\mathcal{K}_{l}^{1 / 2}<+\infty .
\end{aligned}
$$

Thus differentiation under the integral sign holds, the integral (3.8) is $n-l$ times differentiable, and so are the eigenfunctions $\phi_{i}^{[l]}$. An analogous argument shows that the integral corresponding to the $(n-l)$ th derivative under the integral sign is continuous in $I$. Thus eigenfunctions corresponding to nonzero eigenvalues are $C^{n-l}(I)$.

The norm estimates work identically for bounded or unbounded $I$, so from now on we need not make any assumption about it. By the Cauchy-Schwartz inequality and Theorem 2.3 we have

$$
\begin{aligned}
\left\|\phi_{i}^{[l](m-l)}\right\|_{L^{2}(I)}^{2} & =\int_{I}\left|\phi_{i}^{[l](m-l)}(x)\right|^{2} d x \\
& =\int_{I}\left|\frac{1}{\lambda_{i}^{[l]}} \int_{I}\left(\frac{\partial^{m-l}}{\partial x^{m-l}} k_{l}(x, y)\right) \phi_{i}^{[l]}(y) d y\right|^{2} d x \\
& \leq\left(\frac{1}{\lambda_{i}^{[l]}}\right)^{2} \int_{-\infty}^{+\infty}\left[\int_{I}\left|\frac{\partial^{m-l}}{\partial x^{m-l}} k_{l}(x, y)\right|^{2} d y \int_{I}\left|\phi_{i}^{[l]}(y)\right|^{2} d y\right] d x
\end{aligned}
$$




$$
\begin{aligned}
& \leq\left(\frac{1}{\lambda_{i}^{[l]}}\right)^{2} \int_{I}\left[\int_{I} \frac{\partial^{2(m-l)} k_{l}(x, x)}{\partial y^{m-l} \partial x^{m-l}} k_{l}(y, y) d y\right] d x \cdot\left\|\phi_{i}^{[l]}\right\|_{L^{2}(I)}^{2} \\
& =\left(\frac{1}{\lambda_{i}^{[l]}}\right)^{2} \int_{I} k_{m}(x, x) d x \int_{I} k_{l}(y, y) d y=\left(\frac{1}{\lambda_{i}^{[l]}}\right)^{2} \mathcal{K}_{m} \mathcal{K}_{l}
\end{aligned}
$$

for all $0 \leq l \leq m \leq n$ with $l+m \leq n$. Thus

$$
\left\|\phi_{i}^{[l]}\right\|_{H^{n-l}(I)}^{2}=\sum_{m=l}^{n}\left\|\phi_{i}^{[l](m-l)}\right\|_{L^{2}(I)}^{2} \leq\left(\frac{1}{\lambda_{i}^{[l]}}\right)^{2} \sum_{m=l}^{n} \mathscr{K}_{m} \mathcal{K}_{l}
$$

or, recalling definition (3.6), $\left\|\phi_{i}^{[l]}\right\|_{H^{n-l}(I)} \leq C_{n, l} / \lambda_{i}^{[l]}$ as asserted.

Since the operators with kernels $k_{l}$ are compact and positive, for each $l$ the eigenvalue sequence $\left\{\lambda_{i}^{[l]}\right\}_{i \in \mathbb{N}}$ may be assumed to be decreasing to 0 . We denote by $E_{N}^{[l]}=\oplus_{i=1}^{N} E_{\lambda_{i}^{[l]}}$ the direct sum of the eigenspaces associated with the first $N$ eigenvalues of $k_{l}$.

Corollary 3.6. Suppose $k(x, y)$ is a positive definite kernel in class $\mathscr{A}_{n}(I)$ and let $0 \leq l \leq n$. Suppose $\lambda_{N}^{[l]}$ is a nonzero eigenvalue of $k_{l}$. Then for any $\phi \in E_{N}^{[l]}$,

$$
\|\phi\|_{H^{n-l}(I)} \leq C_{n, l}\left[\sum_{i=1}^{N}\left(\frac{1}{\lambda_{i}^{[l]}}\right)^{2}\right]^{1 / 2}\|\phi\|_{L^{2}(I)} .
$$

Proof. Since $\left\{\phi_{i}^{[l]}\right\}_{i=1}^{N}$ constitute an $L^{2}(I)$-orthonormal basis for $E_{N}^{[l]}$, we have $\phi=\sum_{i=1}^{N} c_{i} \phi_{i}^{[l]}$ with $\|\phi\|_{L^{2}(I)}^{2}=\sum_{i=1}^{N}\left|c_{i}\right|^{2}$. For $l \leq m \leq n$,

$$
\begin{aligned}
\left\|\phi^{(m)}\right\|_{L^{2}(I)}^{2} & =\left\|\sum_{i=1}^{N} c_{i} \phi_{i}^{[l](m)}\right\|_{L^{2}(I)}^{2} \leq\left(\sum_{i=1}^{N}\left|c_{i}\right|\left\|\phi_{i}^{[l](m)}\right\|_{L^{2}(I)}\right)^{2} \leq\left(\sum_{i=1}^{N}\left|c_{i}\right|^{2}\right)\left(\sum_{i=1}^{N}\left\|\phi_{i}^{[l](m)}\right\|_{L^{2}(I)}^{2}\right) \\
& \leq\|\phi\|_{L^{2}(I)}^{2} \sum_{i=1}^{N}\left(\frac{1}{\lambda_{i}^{[l]}}\right)^{2} \mathcal{K}_{m} \mathcal{Y}_{l} .
\end{aligned}
$$

Therefore

$$
\begin{aligned}
\|\phi\|_{H^{n-l}(I)} & =\left(\sum_{m=l}^{n}\left\|\phi^{(m)}\right\|_{L^{2}(I)}^{2}\right)^{1 / 2} \leq \mathscr{K}_{l}^{1 / 2}\left(\sum_{m=l}^{n} \mathscr{K}_{m}\right)^{1 / 2}\left[\sum_{i=1}^{N}\left(\frac{1}{\lambda_{i}^{[l]}}\right)^{2}\right]^{1 / 2}\|\phi\|_{L^{2}(I)} \\
& =C_{n, l}\left[\sum_{i=1}^{N}\left(\frac{1}{\lambda_{i}^{[l]}}\right)^{2}\right]^{1 / 2}\|\phi\|_{L^{2}(I)}
\end{aligned}
$$

as stated.

Remark 3.7. The norm bound obtained in (3.7) cannot, in general, be improved. To show this let $I \subset \mathbb{R}$ and choose $\phi \in C^{n-l}(I) \cap H^{n-l}(I)$ with $\|\phi\|_{L^{2}(I)}=1$ and $\phi(x) \rightarrow 0$ as $|x| \rightarrow \infty$ 
if $I$ is unbounded. By Remark 3.2 these choices imply that $k_{l}(x, y)=\phi(x) \overline{\phi(y)}$ is a rank1 positive definite kernel in class $\mathscr{A}_{n-l}(I)$ irrespective of whether $I$ is bounded or not. In particular the only nonzero eigenvalue is $\lambda^{[l]}=1$ and the corresponding normalized eigenvector is $\phi$. Recalling the definition (3.5) of $\mathcal{K}_{m}$, we have in this case

$$
\mathscr{K}_{m}=\int_{I} k_{m}(x, x) d x=\int_{I}\left|\phi^{(m-l)}(x)\right|^{2} d x=\left\|\phi^{(m-l)}\right\|_{L^{2}(I)}^{2}
$$

for $0 \leq l \leq m \leq n$. By our choice of $k_{l}$ we have $\mathscr{K}_{l}=\|\phi\|_{L^{2}(I)}^{2}=1$ and, since $\lambda^{[l]}=1$, we may write

$$
\|\phi\|_{H^{n-l}}^{2}=\sum_{m=l}^{n}\left\|\phi^{(m-l)}\right\|_{L^{2}(I)}^{2}=\sum_{m=l}^{n} \mathscr{K}_{m}=\frac{\mathscr{K}_{l}}{\lambda^{[l]}} \sum_{m=l}^{n} \mathscr{K}_{m},
$$

and so in this case equality holds in (3.11). This shows that the bound in Theorem 3.5 is sharp and cannot be improved.

\section{References}

[1] N. Aronszajn, Theory of reproducing kernels, Transactions of the American Mathematical Society 68 (1950), no. 3, 337-404.

[2] J. Buescu, Positive integral operators in unbounded domains, Journal of Mathematical Analysis and Applications 296 (2004), no. 1, 244-255.

[3] J. Buescu, F. Garcia, I. Lourtie, and A. C. Paixão, Positive-definiteness, integral equations and Fourier transforms, Journal of Integral Equations and Applications 16 (2004), no. 1, 33-52.

[4] J. Buescu and A. C. Paixão, Positive definite matrices and integral equations on unbounded domains, Differential and Integral Equations 19 (2006), no. 2, 189-210.

[5] E. H. Moore, General Analysis. Pt. I, Memoirs of Amer. Philos. Soc., American Philosophical Society, Pennsylvania, 1935.

[6] - General Analysis. Pt. II, Memoirs of Amer. Philos. Soc., American Philosophical Society, Pennsylvania, 1939.

[7] F. Riesz and B. Nagy, Functional Analysis, Ungar, New York, 1952.

[8] S. Saitoh, Theory of Reproducing Kernels and Its Applications, Pitman Research Notes in Mathematics Series, vol. 189, Longman Scientific \& Technical, Harlow, 1988.

Jorge Buescu: Departamento de Matemática, Instituto Superior Técnico, 1049-001 Lisbon, Portugal E-mail address: jbuescu@math.ist.utl.pt

A. C. Paixão: Departamento de Engenharia Mecânica, ISEL, 1949-014 Lisbon, Portugal

E-mail address: apaixao@dem.isel.ipl.pt 